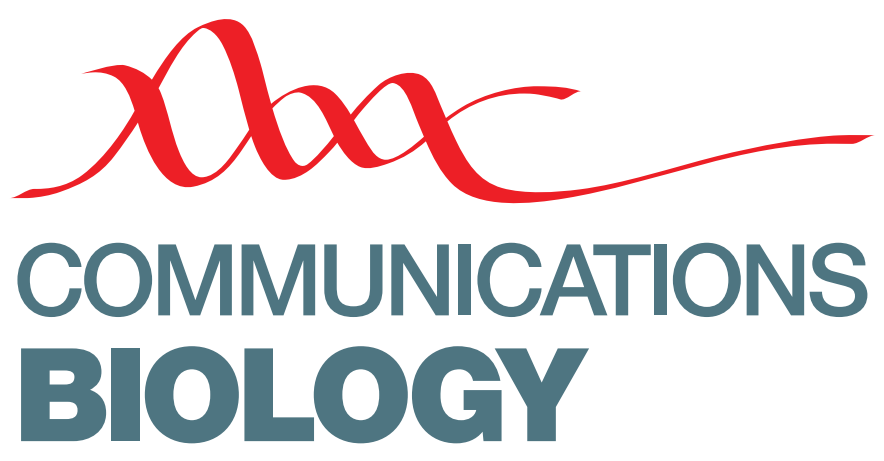

ARTICLE

DOI: $10.1038 / \mathrm{s} 42003-018-0035-5 \quad$ OPEN

\title{
Highly efficient base editing in bacteria using a Cas9-cytidine deaminase fusion
}

Ke Zheng ${ }^{1}$, Yang Wang ${ }^{1}$, Na Li ${ }^{1}$, Fang-Fang Jiang ${ }^{1}$, Chang-Xian $\mathrm{Wu}^{1}$, Fang Liu ${ }^{1}$, Huan-Chun Chen ${ }^{1} \&$ Zheng-Fei Liu ${ }^{1}$

The ability to precisely edit individual bases of bacterial genomes would accelerate the investigation of the function of genes. Here we utilized a nickase Cas9-cytidine deaminase fusion protein to direct the conversion of cytosine to thymine within prokaryotic cells, resulting in high mutagenesis frequencies in Escherichia coli and Brucella melitensis. Our study suggests that CRISPR/Cas9-guided base-editing is a viable alternative approach to generate mutant bacterial strains.

\footnotetext{
${ }^{1}$ State Key Laboratory of Agricultural Microbiology and Key laboratory of Preventive Veterinary Medicine in Hubei Province, College of Veterinary Medicine, Huazhong Agricultural University, Wuhan 430070, China. Correspondence and requests for materials should be addressed to

Z.-F.L. (email: Izf6789@mail.hzau.edu.cn)
} 
$\mathrm{P}$ rogrammable modification of genomes is a vital approach to the study of gene function ${ }^{1-3}$. Recombineering using the recA and Lambda Red systems are prevalent methods for constructing modified strains in various bacteria species ${ }^{4-10}$. RecA-mediated recombination needs long homologous target gene sequences ( $\geq 500 \mathrm{bp})$, and occurs at a low frequency $\left(10^{-6}\right.$ to $\left.10^{-4}\right)^{4,5}$. In contrast, the $\lambda$ Red system shows high recombination efficiency $\left(\sim 10^{-3}\right.$ to $\left.10^{-1}\right)$, and requires as little as $30 \mathrm{bp}$ of homologous sequence to serve as a substrate $e^{6-10}$. Recently, the CRISPR (clustered, regularly interspaced, short palindromic repeats) /Cas9 system ${ }^{1,2}$ was employed to assist editing of bacterial genomes by killing non-edited cells, leading to a recovery of almost $100 \%$ edited cells ${ }^{11,12}$. However, donor DNA as editing template is still required to knock out genes in bacterial cells using these methods.

Recently, a system called "Base Editor" (abbreviated to BE) was reported, which fused cytidine deaminase to a CRISPR-associated Cas9 variant to convert cytosine (C) to uracil (U) at targeted sites without double-strand breaks (DSBs), resulting in $\mathrm{C} \rightarrow \mathrm{T}$ (or $\mathrm{G} \rightarrow$ A) substitution ${ }^{13}$. The base-editing system directly edits single nucleotides, which avoids the dependence on homologydependent repair (HDR). Various derivations of the BE system, such as BE1, BE2 and BE3, have been developed and all the derivations induce base-editing in mammalian cells with very low indel rates. These systems have been applied to mammalian cells, animals and plants ${ }^{13-18}$. Other CRISPR-guided BE systems using human AID (activation-induced cytidine deaminase) ${ }^{19}$ or AID ortholog PmCDA1 from sea lamprey ${ }^{20}$ have been reported and demonstrated in eukaryotic organisms ${ }^{21,22}$. Some researchers used $\mathrm{BE} 3$ to engineer premature coding termination to provide an alternative approach to knock out genes ${ }^{15,18,23}$. We envisioned that a highly efficient BE3 system would enable rapid and efficient programmable editing in bacteria.

In the present work, we constructed bacterial expressing BE3 plasmids that induced the substitution of $\mathrm{C}$ to $\mathrm{T}$ to convert a Gln codon (CAA) to a stop codon (TAA) in the tetA gene conferring tetracycline resistance at close to $100 \%$ efficiency in E. coli strain XL1-Blue (tetracycline insensitive). Next, the lac $Z$ and $r p p H$ genes were selected to further validate the same method in E. coli. We also demonstrated that BE3 can achieve precise and efficient base conversion within Brucella melitensis (B. melitensis) in a targeted manner. Collectively, the BE3 editor was shown to be a powerful tool for direct base changes in E. coli and B. melitensis.

\section{Results}

E. coli genome editing using BE3. First, we tested whether BE3 can function in E. coli cells. The BE3 editing element was cloned into an inducible expression vector, under control of the $\mathrm{PL}_{\text {lacO-1 }}$ promoter (Supplementary Fig. 1, Supplementary Note 1). The E. coli strain XL1-Blue was selected as a model because the Tn 10 transposon containing the tetracycline-resistance gene tetA is located in its genome ${ }^{24}$. We predicted that the CAG/CAA (Gln) or CGA (Arg) codons would be converted into respective TAG/ TAA/TGA premature Stop codons, thereby inactivating the TetA protein tetracycline efflux pump function. The sgRNAs were designed within the first third of the tet $A$ open reading frame to truncate the protein (Fig. 1a). The base-editing occurred during the transformation recovery step when the BE3 protein was expressed and performed its function ( $1 \mathrm{~h}$ post heat shock). After base-editing, the edited E. coli cells were isolated by plate streaking to avoid satellite colony contamination and then grown in liquid cultures. A $2 \mu \mathrm{L}$ volume of each liquid culture was dropped on plates $\left(\geq 2.605 \times 10^{7}\right.$ colony-forming unit (CFU), concentration: $1.3025 \times 10^{10} \mathrm{CFU} \mathrm{mL}^{-1}$, Supplementary Table 1, Supplementary Table 2) to detect the loss of antibiotic resistance (Fig. 1b). For the BE3-induced incapacitating TetA at site 2, all colonies (20/20) showed a loss of function (Fig. 1b). Mutagenesis at both sites was confirmed by PCR amplification and Sanger sequencing. For the sgRNA1-guided mutagenesis, the base substitution occurred at an undesired codon (ATC|Ile to ATT|Ile) rather than at the designed one (Fig. 1c). The sequencing of tetracycline-sensitive colonies showed that the CAA|Gln codon
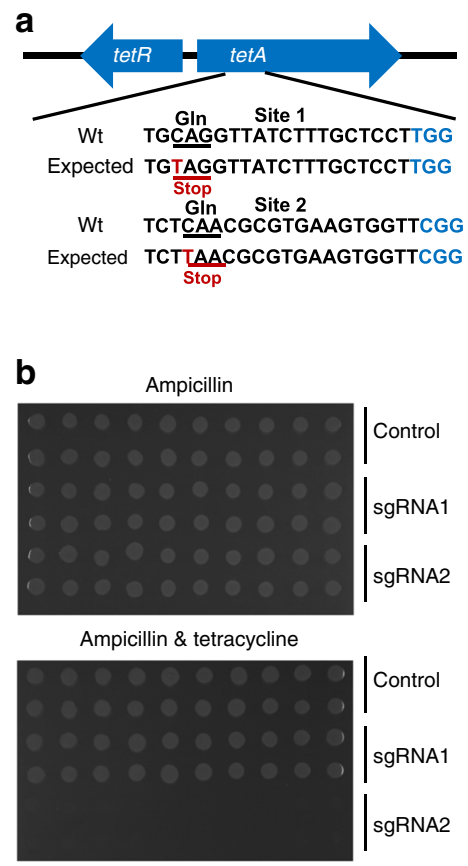
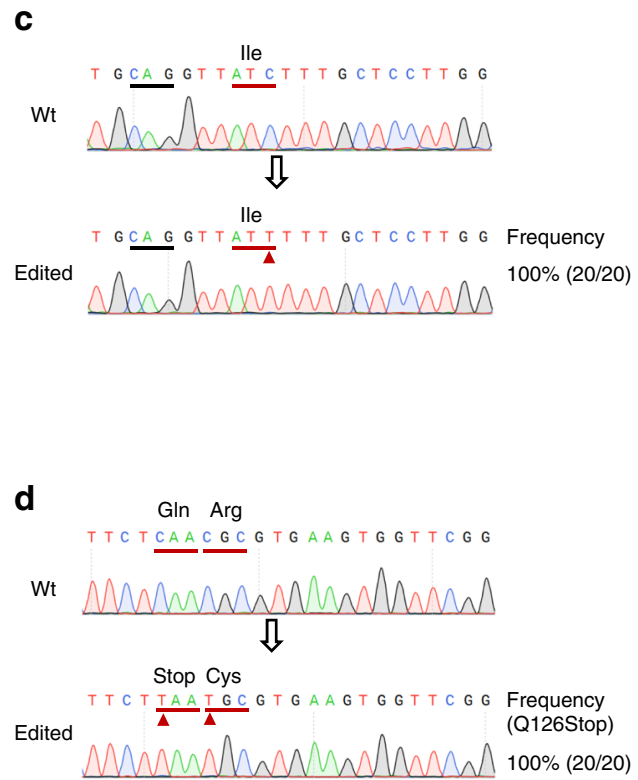

Fig. 1 Induction of base conversion at tetA sites in E. coli. a The designed mutation sites in the tetA gene. PAM motif (blue), target sites (red). b Colony tetracycline-resistance test. c Sanger sequencing at tet $A$ gene site 1. d Sanger sequencing at tet $A$ gene site 2. The substituted bases are marked with a red arrow. The transformations and base-editing assays were repeated for three times. The phenotype assay and Sanger sequencing were performed on 20 colonies of each group. The editing frequency was calculated by formula (edited colony/total colony) 
a

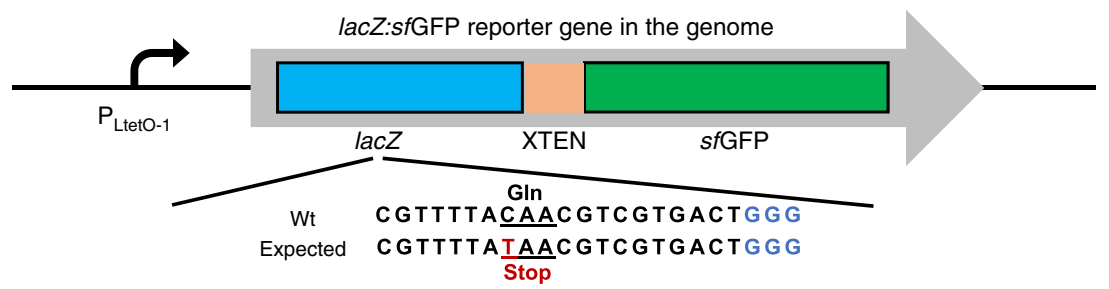

b

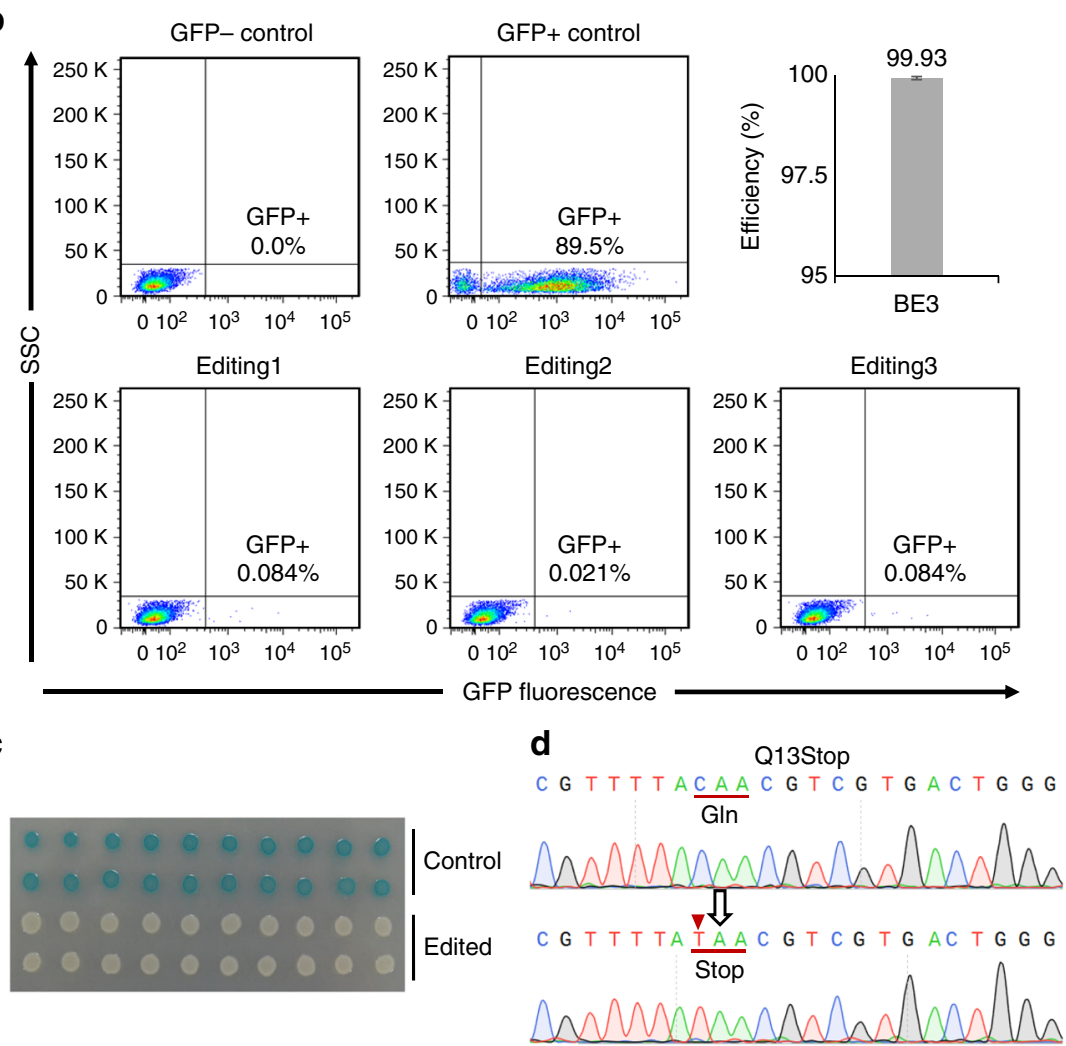

Fig. 2 Highly efficient base-editing in E. coli. a Schematic representation of the lacZ:sfGFP reporter gene in engineered E. coli strain GS1783-lacZ:sfGFP. b Flow cytometry analysis of base-editing efficiency based on GFP fluorescence. The morphological complexity of cells was measured by side scatter light (SSC). Three biological replicates were assayed. E. coli strain GS1783 was used as a GFP-negative control and E. coli strain GS1783-lacZ:sfGFP was used as a GFP-positive $\left(\mathrm{GFP}^{+}\right)$control. The editing efficiency was calculated by formula: editing frequency $=1-\frac{\text { Percentage of GFP }{ }^{+} \text {cells in edited population }}{\text { Percentage of } \mathrm{GFP}^{+} \text {cells in unedited population. }}$. Resulting phenotypes (X-gal reaction indicated LacZ activity) of isolated $E$. coli. $\mathbf{d}$ Sanger sequencing of lacZ:sfGFP gene editing. The substituted base is marked with a red arrow. The transformations and phenotype assays were repeated for three times. The phenotype assay and Sanger sequencing were performed for 20 colonies of each group. The editing frequency was calculated by formula (edited colony/total colony)

was converted to a TAA|Stop codon, and that other codons were also changed by base conversion (Fig. 1d). Editing was $100 \%$ efficient at some sites.

Editing efficiency of BE3 in E. coli. Next, we constructed a lacZ: $s f G F P$ reporter gene-integrated $E$. coli strain to calculate the accuracy of editing efficiency. A DNA fragment containing the $\mathrm{P}_{\text {LtetO-1 }}$ promoter, which controlled the lacZ:sfGFP-fused reporter gene and the chloramphenicol-resistance gene (Fig. 2a), was PCR amplified from pXG-10sf ${ }^{25}$. This fragment harbored homologous sequence and integrated into the genome through $\lambda$ red-mediated recombination. The base-edited colonies were cultured in LB medium and analyzed by flow cytometry and X-gal cytochemistry. Flow cytometry showed that $99.93 \%$ of edited $E$. coli cells lost fluorescence (Fig. 2b), indicating that the BE3-mediated baseediting in $E$. coli was almost $100 \%$ efficient. X-gal reactions and Sanger sequencing confirmed the editing (Fig. 2c, d). We also tested $\mathrm{BE} 3$ on the $r p p H$ gene and produced highly efficient editing
(Fig. 3). In addition, cytotoxicity when using BE3 was measured by transformation. Compared to wtCas9, BE3 protein is nonlethal when it targets and nicks the genome (Supplementary Fig. 2).

B. melitensis genome engineering using BE3. We next applied this editor in B. melitensis, an a proteobacteria and facultative intracellular bacterial pathogen. To apply the base-editing system in B. melitensis, we constructed an IPTG-inducible expression vector based on the broad host-range plasmid, pBBR1-MCS5 ${ }^{26}$, and tested inducing conditions (Fig. 4a). The BE3 gene was cloned into the inducible expression vector under control of the trc promoter (Supplementary Fig. 3, Supplementary Note 2). Three sgRNAs were designed for mutagenesis of the virB10 gene (Fig. 4b). The transformants were cultured in TSB medium, followed by inactivation and genomic DNA extraction. The target regions were amplified and sequenced. The conversion occurred 
a

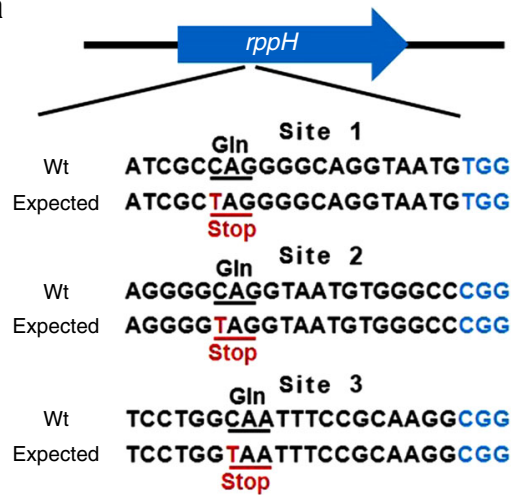

C

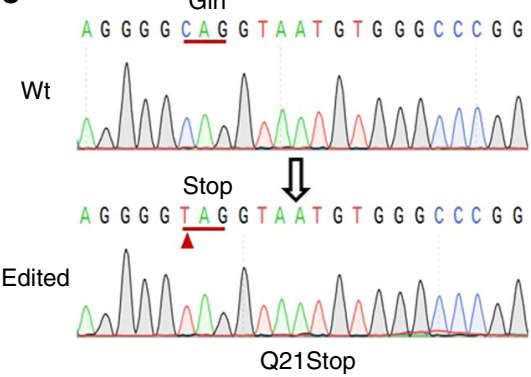

frequency $100 \%(10 / 10)$ b
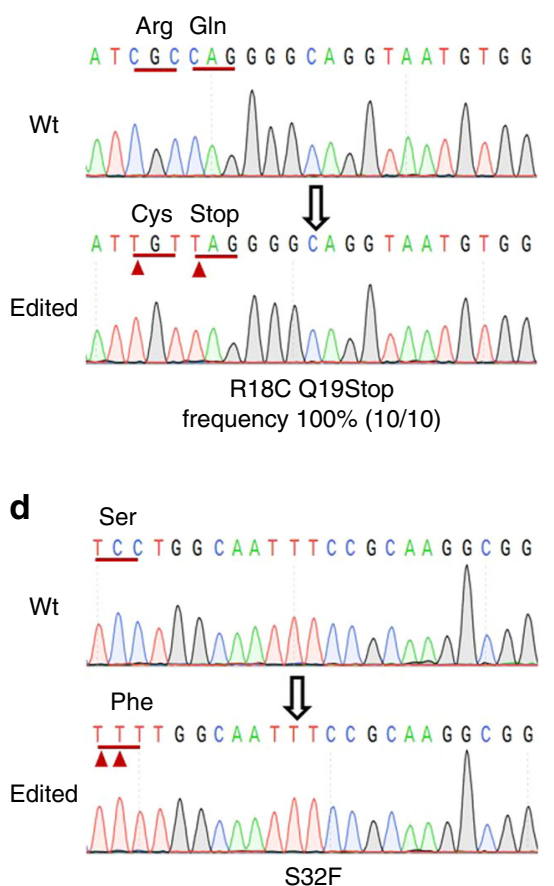

frequency $100 \%(10 / 10)$

Fig. 3 Induction of base conversion at rppH sites in E. coli. a The designed mutation sites in the rppH gene. PAM motif (blue), target sites (red). b c, d Sanger sequencing of base-editing at different loci in the rppH gene. The substituted bases are marked with red arrows. The transformations and base-editing assays were repeated for three times. The phenotype assay and Sanger sequencing were performed on 10 colonies of each group. The editing frequency was calculated by formula (edited colony/total colony)

at only one site among the candidates with $100 \%$ efficiency (Fig. 4c).

We demonstrated that CRISPR/Cas9-guide-specific base conversion could be achieved in Gram-negative bacteria, E. coli and B. melitensis, with high efficiency. The conversion at expected sites resulted in premature termination of coding genes, which abolished protein function. This finding simplifies the process of base mutagenesis and expands the applications of the CRISPR/ Cas9 system in bacterial cells.

\section{Discussion}

Recently, programmable base conversion has been reported in $E$. coli by fusing cytidine deaminase with ZF (zinc finger) or TALE (transcription activator-like effectors)-DNA binding domains ${ }^{27}$. A single copy GFP reporter gene locus possessing a 'broken' start codon ('ACG') was rescued by deamination with up to $13 \%$ efficiency in an uracil repair gene-deleted E. coli strain. In our study, the CRISPR/Cas9-guided BE3 system achieved much higher efficiency because a single-strand DNA ' $\mathrm{R}$ loop' structure $^{28}$, a natural substrate for APOBEC1 deaminase ${ }^{29}$, is generated by Cas 9 unwinding activity ${ }^{28}$. The break on the non-edited strand mediated by nCas 9 enhances the trend towards the expected editing ${ }^{13}$.

Some limitations remain for using base-editing as a way to knock out a gene: first, the premature stop codon depends on CAA/CAG/CGA/TGG codons; second, a truncation site should be at an appropriate location in an ORF; additionally, we should not ignore the fact that the editing efficiency of the BE systems followed the order $\mathrm{TC} \geq \mathrm{CC} \geq \mathrm{AC} \geq \mathrm{GC}^{13}$.

Recently, improved $\mathrm{BE}$ systems have been reported. On the basis of Cas9 homology and engineered variants, improved base editors with different protospacer adjacent motif (PAM) specificities were developed and demonstrated in mammalian cells ${ }^{30}$ and zebrafish ${ }^{31}$. Furthermore, the width of the deamination window can be narrowed to 1-2 nt by engineered APOBEC1 enzymes $^{30}$. Notably, programmable base editing of $A$ to $G$ was reported recently 32 , which broadens the application of base editing. The wild-type E. coli tRNA-specific adenosine deaminase (ecTadA)-dCas9 fused protein with no ability of deaminate adenine at target loci in DNA was transformed into high efficient adenine base editors (ABEs) after a series of evolution and engineering. The ABEs show high efficient A-to-G editing in both E. coli and human cells. The BEs and ABEs enable programmable editing of all four nucleotide without DSBs. Taken together, we show that CRISPR-guided BE3 can perform highly efficient baseediting in bacterial cells, which may reduce the time and efforts that take to manipulate bacterial genes and to obtain viable mutant bacterial strains.

\section{Methods}

Construction of pEcBE3 and pBmBE3 vectors. The original plasmid containing the BE3 element (rAPOBEC1-XTEN-Cas9n-UGI-NLS) was obtained from Addgene (pCMV-BE3, \#73021) ${ }^{13}$. The BsaI restriction sites in the BE3 element were removed through site-directed mutagenesis by Gibson assembly. The mutated BE3 was cloned into the Pml I site in the inducible expression vector pZF17-31 (Supplementary Fig. 3, Supplementary Note 2) by Gibson assembly, resulting in pZF17-32 (Supplementary Fig. 4, Supplementary Note 3). The sgRNA gene was amplified from pZF17-33 (Supplementary Fig. 5, Supplementary Note 4) and cloned into the XhoI site in pZF17-32, resulting in inducible expression vector pEcBE3 (Supplementary Fig. 1, Supplementary Note 1) for use in base editing in $E$. coli. The pBmCRISPR2 vector (Supplementary Fig. 6, Supplementary Note 5) used for expressing Cas 9 and sgRNA in B. melitensis was used as the vector backbone for $\mathrm{pBmBE3}$. The Cas9 coding sequence was replaced by the mutated BE3 element through Gibson assembly, to obtain pBmBE3 (Supplementary Fig. 7, Supplementary Note 6). For sgRNA cloning, synthesized oligonucleotides were annealed to form a dimer, which was then ligated into BsaI-digested $\mathrm{pEcBE} 3$ or $\mathrm{pBmBE} 3$. PCR was performed using Phanta Max Super-Fidelity DNA Polymerase (Vazyme), and Gibson assembly was performed according to a reported protocol ${ }^{33}$. Oligonucleotide sequences are listed in Supplementary Table 3-4. 
a
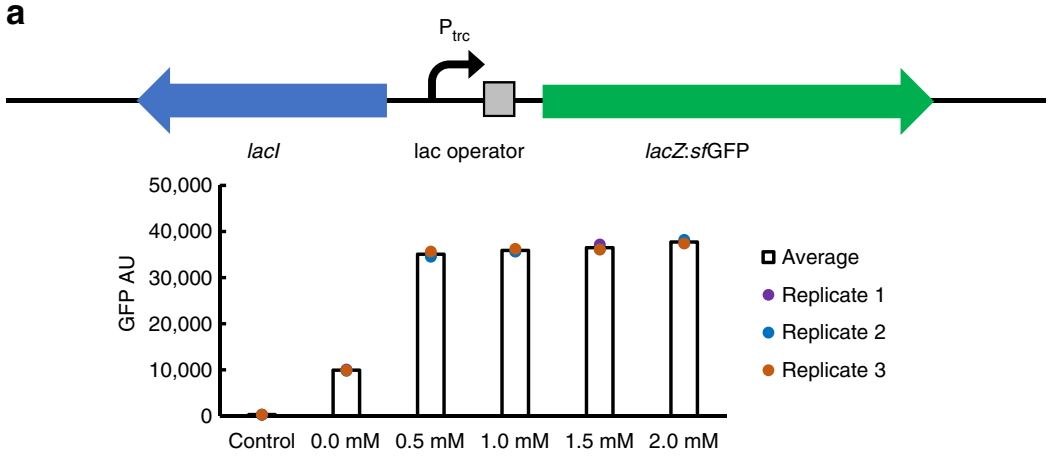

IPTG

b

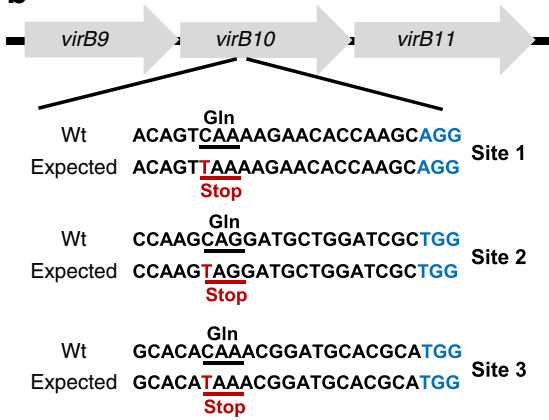

C

Gln

ACAGT CAAAAGACACCAAGCAGG

Wt

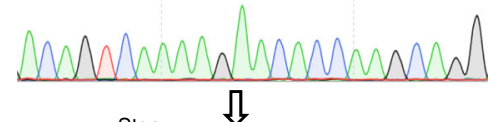

Stop

ACAGT TAAAAGACACCAAGCAGG

Edited

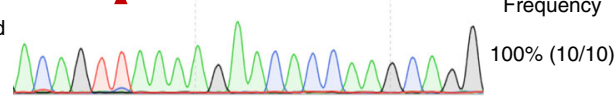

Fig. 4 Premature stop codon generated by the BE3 system in B. melitensis. a Expression levels of lacZ:sfGFP from the pZK79-lacZ:sfGFP vector under repressed and induced conditions. b The designed mutation sites in the virB10 gene. PAM motif (blue), target sites (red). c Sanger sequencing at virB10 gene site 1. The substituted base is marked with a red arrow. The transformations and base-editing assays were repeated for three times. The phenotype assay and Sanger sequencing were performed on 10 colonies of each group. The editing frequency was calculated by formula (edited colony/total colony)

Base-editing assay and screening. We performed the base-editing assay during the transformation recovery step. Chemically competent $E$. coli cells were transformed with pEcBE3 series plasmids. After heat-shock, transformed E. coli cells were incubated in SOC medium (containing $0.6 \mathrm{mM} \mathrm{IPTG)} \mathrm{at} 37^{\circ} \mathrm{C}$ shaking at 180 r.p.m for $1 \mathrm{~h}$. Cells were then spread on LB agar plates (containing $50 \mu \mathrm{g} \mathrm{mL} \mathrm{L}^{-1}$ ampicillin and $0.6 \mathrm{mM}$ IPTG). For mutagenesis in B. melitensis, pBmBE3 series plasmids were transformed into strain $16 \mathrm{M}$ electro-competent cells by electroporation. The transformed cells were recovered in TSB (Tryptic Soy Broth, BD) medium (containing $0.6 \mathrm{mM} \mathrm{IPTG)}$ at $37^{\circ} \mathrm{C}$ shaking at $180 \mathrm{r} . \mathrm{p} . \mathrm{m}$. for $4 \mathrm{~h}$ and were then spread on TSA plates (containing $50 \mu \mathrm{g} \mathrm{mL}^{-1}$ gentamycin and $0.6 \mathrm{mM}$ IPTG). Empty BE3 vectors carrying non-targeting sgRNA were transformed as control. Single colonies were then cultured in LB medium (for E. coli) or TSB medium (for B. melitensis) and genomic DNA extracted using a bacterial genome DNA extraction kit (TIANGEN). Since satellite colonies occurs when the ampicillinresistance gene contained in pEcBE3 series plasmids is used, it is necessary to isolate the transformed E. coli colonies so as to avoid contamination being contaminated by satellite colonies. For each plate, the colonies were resuspended in 1 $\mathrm{mL}$ of $\mathrm{LB}$ medium, and spreaded on LB agar plate (containing $50 \mu \mathrm{g} \mathrm{mL}^{-1}$ ampicillin and $0.6 \mathrm{mM}$ IPTG) using sterile incubation loop according to T-Streak methods. The plate then was incubated at $37^{\circ} \mathrm{C}$ overnight to obtain single colony. The target regions were amplified by PCR and sequenced to confirm mutagenesis. The mineral oil was added to PCR tube to avoid aerosol contamination. The primers used are listed in Supplementary Table 3.

E. coli reporter strain construct. The lacZ:sfGFP gene was selected as a reporter gene. The lacZ:sfGFP-fused gene and chloramphenicol-resistance gene $\left(\mathrm{Cm}^{\mathrm{R}}\right)$ were amplified from pXG-10sf ${ }^{25}$ using two cycle PCR, which harbored homologous sequence targeting the E. coli strain GS1783 $3^{34}$ genome. GS1783 cells were prepared as electro-competent cells according to a previous study ${ }^{34}$. The gel-purified PCR product was electro-transformed into GS1783 cells. After incubation in $1 \mathrm{~mL} \mathrm{SOC}$ medium at $37^{\circ} \mathrm{C}$ with shaking at 180 r.p.m., the transformed cells were screened on $\mathrm{LB}$ agar plates containing $34 \mu \mathrm{g} \mathrm{mL}^{-1}$ chloramphenicol. The primers used are listed in Supplementary Table 3.

Inducing conditions of the trc promoter in B. melitensis. The lacZ:sfGFP gene was cloned into the NsiI and $\mathrm{XbaI}$ sites of the inducible expression vector, pZK79 (Supplementary Fig. 8, Supplementary Note 7, in previous study not reported), to obtain pZK79-lacZ:sfGFP. The pZK79 and pZK79-lacZ:sfGFP were electrotransformed into $B$. melitensis $16 \mathrm{M}$ competent cells, respectively. Transformants were cultured in $5 \mathrm{~mL}$ TSB medium $\left(50 \mu \mathrm{g} \mathrm{mL}^{-1}\right.$ gentamycin) for $48 \mathrm{~h}$, and then in fresh $5 \mathrm{~mL}$ TSB medium $\left(50 \mu \mathrm{g} \mathrm{mL}^{-1}\right.$ gentamycin) at a ratio of 1:50 until the $\mathrm{OD}_{600}$ reached $\sim 0.6$. IPTG was then added to gradient final concentration $(0 \mathrm{mM}$, $0.5 \mathrm{mM}, 1.0 \mathrm{mM}, 1.5 \mathrm{mM}$ and $2.0 \mathrm{mM}$ ) and the culture continued for $6 \mathrm{~h}$. Phenol was then added at a final concentration of $1 \%(\mathrm{v} / \mathrm{v})$ to inactivate the culture. Cells were then collected and washed with PBS (phosphate-buffered saline buffer). GFP fluorescence was measured using a PerkinElmer EnVision plate reader (the $\mathrm{OD}_{600}$ value was used to normalize cell concentration). Primers used are listed in Supplementary Table 3.

Phenotype assay. Isolated single colonies were cultured in LB medium $(50 \mu \mathrm{g} \mathrm{mL}$ ${ }^{-1}$ ampicillin) and $2 \mu \mathrm{L}$ of bacterial culture then spread on LB agar plates. In the LacZ activity assay, we used ampicillin $\left(50 \mu \mathrm{g} \mathrm{mL}^{-1}\right)$ and X-gal $\left(40 \mu \mathrm{g} \mathrm{mL}^{-1}\right) \mathrm{LB}$ agar plates. In the tetracycline-sensitive assay, we used ampicillin $\left(50 \mu \mathrm{g} \mathrm{mL}^{-1}\right)$ or ampicillin $\left(50 \mu \mathrm{g} \mathrm{mL}^{-1}\right)$ plus tetracycline $\left(10 \mu \mathrm{g} \mathrm{mL}^{-1}\right) \mathrm{LB}$ agar plates. Bacterial culture concentration was assessed by optical density $\left(\mathrm{OD}_{600}\right)$ measurement and serial dilution-plate count. We measured the $\mathrm{OD}_{600}$ value of 20 bacterial cultures (tetA-editing), and selected the culture with the lowest value for the serial dilutionplate count.

Flow cytometry assay. Transformed GS1783-LacZ:sfGFP cells were resuspended in $2 \mathrm{~mL}$ PBS buffer, and diluted 100 -fold. The diluted cells were analyzed using a BD FACSVerse flow cytometer. The GS1783 cells and untransformed GS1783LacZ:sfGFP cells were taken as GFP-negative and GFP-positive control cells, respectively.

Cytotoxicity assay of BE3. To compare cytotoxicity of wtCas9 and BE3 on E. coli cells, the plasmid pZK77 (Supplementary Fig. 9, Supplementary Note 8) expressing wtCas9 and sgRNA was constructed based on pwtCas9-bacteria (Addgene: \#44250) by inserting a synthesized sgRNA gene into the AvrII site. The pZK77::RppH site was constructed using the same method as for $\mathrm{pEcBE3} 3: \mathrm{RppH}$. DH5a chemically competent cells (Shanghai Weidi Biotechnology) were transformed with $100 \mathrm{ng}$ of pEcBE3::RppH, pEcBE3, pZK77::RppH or pZK77. The empty non-targeted pEcBE3 and pZK77 were controls. After heat-shock, cells were recovered in $1 \mathrm{~mL}$ SOC medium for $1 \mathrm{~h}$ at $37^{\circ} \mathrm{C}$ with shaking at 180 r.p.m. The transformed cells were diluted $10^{3}-10^{5}$-fold or not diluted (for pZK77::RppH) and plated onto Amp LB agar plates.

Statistical analysis. The $t$ test was used to analyze the difference between experiments. 
Data availability. All the data generated or analyzed during this study are included in this published article (and its supplementary information files).

Received: 7 September 2017 Accepted: 21 March 2018

Published online: 19 April 2018

\section{References}

1. Jinek, M. et al. A programmable dual-RNA-guided DNA endonuclease in adaptive bacterial immunity. Science 337, 816-821 (2012).

2. Gasiunas, G., Barrangou, R., Horvath, P. \& Siksnys, V. Cas9-crRNA ribonucleoprotein complex mediates specific DNA cleavage for adaptive immunity in bacteria. Proc. Natl Acad. Sci. USA 109, E2579-E2586 (2012).

3. Wang, H., La Russa, M. \& Qi, L. S. CRISPR/Cas9 in genome editing and beyond. Annu. Rev. Biochem. 85, 227-264 (2016).

4. Sander, P., Meier, A. \& Bottger, E. C. rpsL+: a dominant selectable marker for gene replacement in mycobacteria. Mol. Microbiol. 16, 991-1000 (1995).

5. Pelicic, V., Reyrat, J. M. \& Gicquel, B. Generation of unmarked directed mutations in mycobacteria, using sucrose counter-selectable suicide vectors. Mol. Microbiol. 20, 919-925 (1996).

6. Court, D. L., Sawitzke, J. A. \& Thomason, L. C. Genetic engineering using homologous recombination. Annu. Rev. Genet. 36, 361-388 (2002).

7. Zhang, Y., Buchholz, F., Muyrers, J. P. \& Stewart, A. F. A new logic for DNA engineering using recombination in Escherichia coli. Nat. Genet. 20, 123-128 (1998).

8. Lee, E. C. et al. A highly efficient Escherichia coli-based chromosome engineering system adapted for recombinogenic targeting and subcloning of BAC DNA. Genomics 73, 56-65 (2001).

9. Yu, D. et al. An efficient recombination system for chromosome engineering in Escherichia coli. Proc. Natl Acad. Sci. USA 97, 5978-5983 (2000).

10. Sharan, S. K., Thomason, L. C., Kuznetsov, S. G. \& Court, D. L. Recombineering: a homologous recombination-based method of genetic engineering. Nat. Protoc. 4, 206-223 (2009).

11. Chen, W., Zhang, Y., Yeo, W. S., Bae, T. \& Ji, Q. Rapid and efficient genome editing in staphylococcus aureus by using an engineered CRISPR/Cas9 system. J. Am. Chem. Soc. 139, 3790-3795 (2017).

12. Jiang, W., Bikard, D., Cox, D., Zhang, F. \& Marraffini, L. A. RNA-guided editing of bacterial genomes using CRISPR-Cas systems. Nat. Biotechnol. 31, 233-239 (2013).

13. Komor, A. C., Kim, Y. B., Packer, M. S., Zuris, J. A. \& Liu, D. R. Programmable editing of a target base in genomic DNA without doublestranded DNA cleavage. Nature 533, 420-424 (2016).

14. Rees, H. A. et al. Improving the DNA specificity and applicability of base editing through protein engineering and protein delivery. Nat. Commun. 8, 15790 (2017)

15. Kim, K. et al. Highly efficient RNA-guided base editing in mouse embryos. Nat. Biotechnol. 35, 435-437 (2017).

16. Li, J., Sun, Y., Du, J., Zhao, Y. \& Xia, L. Generation of targeted point mutations in rice by a modified CRISPR/Cas9 system. Mol. Plant 10, 526-529 (2017).

17. Zong, Y. et al. Precise base editing in rice, wheat and maize with a Cas9cytidine deaminase fusion. Nat. Biotechnol. 35, 438-440 (2017).

18. Kuscu, C. et al. CRISPR-STOP: gene silencing through base-editing-induced nonsense mutations. Nat. Methods 14, 710-712 (2017).

19. Ma, Y. et al. Targeted AID-mediated mutagenesis (TAM) enables efficient genomic diversification in mammalian cells. Nat. Methods 13, 1029-1035 (2016).

20. Nishida, K. et al. Targeted nucleotide editing using hybrid prokaryotic and vertebrate adaptive immune systems. Science 353, aaf8729 (2016).

21. Shimatani, Z. et al. Targeted base editing in rice and tomato using a CRISPRCas9 cytidine deaminase fusion. Nat. Biotechnol. 35, 441-443 (2017).

22. Hess, G. et al. Directed evolution using dCas9-targeted somatic hypermutation in mammalian cells. Nat. Methods 13, 1036-1042 (2016).

23. Billon, P. et al. CRISPR-mediated base editing enables efficient disruption of eukaryotic genes through induction of STOP codons. Mol. Cell. 67, 1068-1079 (2017).

24. Hillen, W. \& Schollmeier, K. Nucleotide sequence of the Tn10 encoded tetracycline resistance gene. Nucleic Acids Res. 11, 525-540 (1983).

25. Corcoran, C. P. et al. Superfolder GFP reporters validate diverse new mRNA targets of the classic porin regulator, MicF RNA. Mol. Microbiol. 84, 428-445 (2012).
26. Kovach, M. E. et al. Four new derivatives of the broad-host-range cloning vector pBBR1MCS, carrying different antibiotic-resistance cassettes. Gene $\mathbf{1 6 6}$ 175-176 (1995)

27. Yang, L. et al. Engineering and optimising deaminase fusions for genome editing. Nat. Commun. 7, 13330 (2016).

28. Jiang, F. et al. Structures of a CRISPR-Cas9 R-loop complex primed for DNA cleavage. Science 351, 867-871 (2016).

29. Harris, R. S., Petersen-Mahrt, S. K. \& Neuberger, M. S. RNA editing enzyme APOBEC1 and some of its homologs can act as DNA mutators. Mol. Cell 10, 1247-1253 (2002)

30. Kim, Y. B. et al. Increasing the genome-targeting scope and precision of base editing with engineered Cas9-cytidine deaminase fusions. Nat. Biotechnol. 35, 371-376 (2017).

31. Zhang, Y. et al. Programmable base editing of zebrafish genome using a modified CRISPR-Cas9 system. Nat. Commun. 8, 118 (2017).

32. Gaudelli, N. M. et al. Programmable base editing of $A^{*} T$ to $G^{*} C$ in genomic DNA without DNA cleavage. Nature 551, 464-471 (2017).

33. Gibson, D. G. et al. Enzymatic assembly of DNA molecules up to several hundred kilobases. Nat. Methods 6, 343-345 (2009).

34. Tischer, B. K., Smith, G. A. \& Osterrieder, N. En passant mutagenesis: a two step markerless red recombination system. Methods Mol. Biol. 634, 421-430 (2010).

\section{Acknowledgements}

We would like to thank Dr. Zeliang Chen (Shenyang Agricultural University) for his generous gift of B. melitensis strain $16 \mathrm{M}$. We also thank Dr. Jeremy Allen (Liwen Bianji, Edanz Group China) for editing the English text of this manuscript critically. This work was supported by the National Key Research and Development Program (2016YFD0500105), the Natural Science Foundation of China (31470259 and 31770191), the Technique Innovation Program of Hubei Province (2016ABA124), and Fundamental Research Funds for the Central Universities (2016PY052) to Z-F.L.

\section{Author contributions}

Z-F.L. and K.Z. designed the experiments; K.Z., Y.W., N.L., and F-F.J. performed the experiments on E. coli; K.Z., C-X.W., and F.L. performed the experiments on B. melitensis; H-C.C. provided the B. melitensis strain and reagents; K.Z., and Z-F.L. wrote the manuscript.

\section{Additional information}

Supplementary information accompanies this paper at https://doi.org/10.1038/s42003018-0035-5

Competing interests: The authors declare no competing interests.

Reprints and permission information is available online at http://npg.nature.com/ reprintsandpermissions/

Publisher's note: Springer Nature remains neutral with regard to jurisdictional claims in published maps and institutional affiliations.

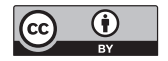

Open Access This article is licensed under a Creative Commons Attribution 4.0 International License, which permits use, sharing, adaptation, distribution and reproduction in any medium or format, as long as you give appropriate credit to the original author(s) and the source, provide a link to the Creative Commons license, and indicate if changes were made. The images or other third party material in this article are included in the article's Creative Commons license, unless indicated otherwise in a credit line to the material. If material is not included in the article's Creative Commons license and your intended use is not permitted by statutory regulation or exceeds the permitted use, you will need to obtain permission directly from the copyright holder. To view a copy of this license, visit http://creativecommons.org/ licenses/by/4.0/.

(C) The Author(s) 2018 